\title{
Creation of an Upper Stage Trajectory Capability Boundary to Enable Booster System Trade Space Exploration
}

\author{
Patrick Walsh ${ }^{\mathrm{i}}$, Adam Coulon ${ }^{\mathrm{ii}}$, Stephen Edwards ${ }^{\mathrm{iii}}$, Dimitri N. Mavris ${ }^{\mathrm{iv}}$ \\ Aerospace Systems Design Laboratory, Georgia Institute of Technology, Atlanta, GA 30332
}

$\mathrm{T}$ here are two approaches which can be taken in launch vehicle analysis and design. The first approach, focused on performance analysis, corresponds to calculating a specific vehicle's payload to orbit capabilities. This can be done in early design, but it is also applicable to studies aimed at evaluating the potential impact of incorporating new technologies onto existing vehicles. The second approach focuses on vehicle sizing, in which the size and mass of a vehicle required to place a specific payload into orbit is calculated. This approach is more common in preconceptual and conceptual design, as payload to orbit capability is typically a driving requirement. For both approaches, trajectory optimization is the critical analysis, with other disciplines such as propulsion and aerodynamics providing the data necessary to model the vehicle.

The problem of trajectory optimization is important in all space missions. The solution of this problem enables one to specify the optimum thrust steering program which should be followed to achieve a specified mission objective, simultaneously satisfying the constraints. ${ }^{1}$ It is well known that whether or not the ascent trajectory is optimal can have a significant impact on propellant usage for a given payload, or on payload weight for the same gross vehicle weight. ${ }^{2}$ Consequently, ascent guidance commands are usually optimized in some fashion. Multistage vehicles add complexity to this analysis process as changes in vehicle properties in one stage propagate to the other stages through gear ratios and changes in the optimal trajectory. These effects can cause an increase in analysis time as more variables are added and convergence of the optimizer to system closure requires more analysis iterations.

In this paper, an approach to simplifying this multi-stage problem through the creation of an upper stage capability boundary is presented. This work was completed as part of a larger study focused on trade space exploration for the advanced booster system that will eventually form a part of NASA's new Space Launch System. ${ }^{3}$ The approach developed leverages Design of Experiments and Surrogate Modeling ${ }^{4}$ techniques to create a predictive model of the SLS upper stage performance. The design of the SLS core stages is considered fixed for the purposes of this study, which results in trajectory parameters such as staging conditions being the only variables relevant to the upper stage. Through the creation of a surrogate model, which takes staging conditions as inputs and predicts the payload mass delivered by the SLS upper stage to a reference orbit as the response, it is possible to identify a "surface" of staging conditions which all satisfy the SLS requirement of placing 130 metric tons into low-Earth orbit (LEO). ${ }^{3}$ This identified surface represents the 130 metric ton capability boundary for the upper stage, such that if the combined first stage and boosters can achieve any one staging point on that surface, then the design is identified as feasible. With the surrogate model created, design and analysis of advanced booster concepts is streamlined, as optimization of the upper stage trajectory is no longer required in every design loop.

\section{References}

1. Vathsal, S., Menon, K.A.P., and Swaminathan, R., "Minimax Approach to Trajectory Optimization of Multistage Launch Vehicles," IEEE Transactions on Aerospace and Electronic Systems, Vol. AES-13, No. 2, Pg. 179-187, March 1977.

2. Lu, P., Sun, H., and Tsai, B., "Closed-Loop Endoatmospheric Ascent Guidance,” Journal of Guidance, Control, and Dynamics, Vol. 26, No. 2, March-April 2003.

\footnotetext{
${ }^{\text {i }}$ Graduate Research Assistant, School of Aerospace Engineering, 270 Ferst Dr, Mail Stop 0150, AIAA Member.

ii Graduate Research Assistant, School of Aerospace Engineering, 270 Ferst Dr, Mail Stop 0150, AIAA Member.

iii Research Engineer, School of Aerospace Engineering, 270 Ferst Dr, Mail Stop 0150, AIAA Member.

${ }^{\text {iv }}$ Boeing Professor of Advanced Aerospace Systems Analysis, School of Aerospace Engineering, 270 Ferst Dr, Mail Stop 0150, AIAA Fellow.
} 
3. NASA, "Preliminary Report Regarding NASA's Space Launch System and Multi-Purpose Crew Vehicle," January 2011.

4. Myers, Raymond H., Douglas C. Montgomery, and Christine M. Anderson-Cook, "Response Surface Methodology: Process and Product Optimization Using Designed Experiments," 3rd ed., New Jersey, Wiley, 2009, Print. 